\title{
Which One is Better? Comparison of the Acute Inflammatory Response, Raja Isteri Pengiran Anak Saleha Appendicitis and Alvarado Scoring Systems
}

\author{
Mohammad Yasin Karami, Hadi Niakan ${ }^{1}$, Navid Zadebagheri, Parviz Mardani ${ }^{2}$, Zahra Shayan $^{3}$, \\ Iman Deilami \\ Student Research Committee, Faculty of Medicine, Shiraz University of Medical Sciences, Shiraz; ${ }^{1}$ Trauma Research Center, Shahid Rajaee \\ (Emtiaz) Trauma Hospital, Shiraz University of Medical Sciences, Fars Province, Shiraz; ${ }^{2}$ Department of Surgery, Faculty of Medicine, Shiraz \\ University of Medical Sciences, Shiraz; ${ }^{3}$ Trauma Research Center, Department of Community Medicine, The Medical School, Shiraz University \\ of Medical Sciences, Shiraz, Iran
}

Purpose: Acute appendicitis (AA) is one of the most common causes of an acute abdomen. The accuracies of the Alvarado and the acute inflammatory response (AIR) scores in the diagnosis of appendicitis is very low in Asian populations, so a new scoring system, the Raja Isteri Pengiran Anak Saleha Appendicitis (RIPASA) system, was designed recently. We applied and compared the Alvarado, AIR, and RIPASA scores in the diagnoses of appendicitis in the Iranian population.

Methods: We prospectively compared the RIPASA, Alvarado, and AIR systems by applying them to 100 patients. All the scores were calculated for patients who presented with right quadrant pain. Appendectomies were performed; then, the postoperative pathology reports were correlated with the scores. Scores of 8, 7, and 5 or more are optimal cutoffs for the RIPASA, Alvarado, and AIR scoring systems, respectively. The sensitivities, specificities, positive predictive values, negative predictive values (NPVs), positive and negative likelihood ratios (LRs) for the 3 systems were determined.

Results: The sensitivity and the specificity of the RIPASA score were $93.18 \%$ and $91.67 \%$, respectively. The sensitivities of the Alvarado and the AIR scores were both 78.41\%. The specificities of the Alvarado and the AIR scores were $100 \%$ and $91.67 \%$, respectively. The RIPASA score correctly classified $93 \%$ of all patients confirmed with histological AA compared with $78.41 \%$ for the Alvarado and the AIR scores.

Conclusion: The RIPASA scoring system had more sensitivity, better NPV, a positive LR, and a less negative LR for the Iranian population whereas the Alvarado scoring system was more specific.

Keywords: Alvarado; Raja Isteri Pengiran Anak Saleha Appendicitis; Acute inflammatory response; Appendicitis

\section{INTRODUCTION}

Acute appendicitis (AA) is the most common surgical abdominal emergency diagnosis, occurring in $10 \%$ of the general population;

Received: January 7,2017 • Accepted: July 12, 2017

Correspondence to: Hadi Niakan, M.D.

Trauma Research Center, Rajaei (Emtiaz) Trauma Hospital, Faculty of Medicine, Shiraz University of Medical Sciences, Shiraz, Iran

Tel: +989171800710, Fax: +987136254206, E-mail: karamiy@sums.ac.ir

(C) 2017 The Korean Society of Coloproctology

This is an open-access article distributed under the terms of the Creative Commons Attribution NonCommercial License (http://creativecommons.org/licenses/by-nc/4.0) which permits unrestricted noncommercial use, distribution, and reproduction in any medium, provided the original work is properly cited. nevertheless, it remains a diagnostic problem $[1,2]$. Several clinical indices and laboratory diagnostic tests can lead to an exact diagnosis [3], which is of significant value in choosing the appropriate clinical and surgical treatment [4]. Recently, the Alvarado, the Raja Isteri Pengiran Anak Saleha Appendicitis (RIPASA), and the acute inflammatory response (AIR) scoring systems have been the more common clinical diagnostic scoring systems used for the diagnosis of AA [5-8].

The Alvarado scoring system, which is based on definite clinical findings observed in AA, was advanced to help AA diagnosis and has been revealed to have a suitable diagnostic power [5]. The Alvarado score is broadly used in the diagnosis of AA due to its accessibility and low cost; it also avoids exposing the patient to the 
radiation present in computed tomography (CT). A new scoring system, the RIPASA scoring system, which has more sensitivity and specificity than the Alvarado scoring system, was recently established. On the other hand, the AIR scoring system consists of clinical criteria and two simple laboratory tests, the C-reactive protein (CRP) and the complete blood count tests, and can lead to a better and faster diagnosis of AA [9-13]. Presently, the histopathology study is the gold standard for AA diagnosis. For that reason, we designed this prospective cohort study to compare the diagnoses based on the Alvarado, AIR and RIPASA scoring systems with those obtained from histopathology and to evaluate several predictive diagnostic values.

\section{METHODS}

This prospective cohort study was conducted at Nemazee Hospital, Shiraz, Iran. Ethical Permission in accordance with Interna-

Table 1. Raja Isteri Pengiran Anak Saleha Appendicitis scoring system

\begin{tabular}{|c|c|}
\hline Variable & Score \\
\hline \multicolumn{2}{|l|}{ Sex } \\
\hline Female & 0.5 \\
\hline Male & 1 \\
\hline \multicolumn{2}{|l|}{ Age (yr) } \\
\hline$\leq 40$ & 1 \\
\hline$>40$ & 0.5 \\
\hline Foreign national registration identity card & 1 \\
\hline \multicolumn{2}{|l|}{ Symptoms } \\
\hline RIF pain & 1 \\
\hline Pain migration to RIF & 0.5 \\
\hline Anorexia & 1 \\
\hline Nausea \& vomiting & 1 \\
\hline \multicolumn{2}{|l|}{ Duration of symptoms (hr) } \\
\hline$<48$ & 1 \\
\hline$\geq 48$ & 0.5 \\
\hline \multicolumn{2}{|l|}{ Signs } \\
\hline RIF tenderness & 1 \\
\hline Guarding & 2 \\
\hline Rebound tenderness & 1 \\
\hline Rovsing sign & 2 \\
\hline Temperature between $37^{\circ} \mathrm{C}$ and $39^{\circ} \mathrm{C}$ & 1 \\
\hline \multicolumn{2}{|l|}{ Labs } \\
\hline Elevated white blood cell count & 1 \\
\hline Negative urine analysis (absence of blood, WBCs, bacteria) & 1 \\
\hline Total & 17.5 \\
\hline
\end{tabular}

RIF, right iliac fossa; WBC, white blood cell. tional Helsinki protocols was obtained prior to the beginning of this study. Informed consent was obtained from all patients. The study population included all patients above 18 years old who developed right lower quadrant pain during the period from November 2014 to November 2015 and who were seen at Nemazee Hospital. Pregnant women, patients who had a right iliac fossa mass, patients who did not provide informed consent, patients who had a previous history of urolithiasis and/or pelvic inflammatory disease, and children below 18 years of age were excluded from the study. The sample size necessary for sensitivity $=70 \%$, specificity $=70 \%$, power $=90 \%$, confidence level $=95 \%$, and prevalence of $\mathrm{AA}=70 \%$ was calculated as 100 .

A total of 100 patients were qualified for the study during the study period. The ages of the patients ranged from 18 to 60 years. All 100 patients were scored using the AIR, Alvarado, and RIPASA scoring systems. The Alvarado score contains 8 parameters whereas the RIPASA score contains 18 parameters and the AIR score consists of 5 parameters. The scores for each of the parameters ranged from 0.5 to 2 for the RIPASA system, 1 to 2 for the Alvarado system, and 1 to 3 for the AIR system, as shown in Tables 1,2 , and 3, respectively. Scoring charts were completed by a senior resident on presentation. A score of more than 7 was taken as a high probability of AA for the Alvarado scoring system whereas those scores for the RIPASA and the AIR scoring systems were more than 8 and more than 4, respectively. The positive (PPVs) and negative predictive values (NPVs) and the positive (PLRs) and negative likelihood ratios (NLRs) of the three scoring systems were assessed.

The patients were monitored from the time of admission until discharge from the hospital. Daily follow-up included the monitoring of vital signs twice a day and systemic examination once a

Table 2. Alvarado appendicitis scoring system

\begin{tabular}{lc}
\hline Diagnosis & Score \\
\hline Symptoms & 1 \\
Pain migration to RIF & 1 \\
Anorexia & 1 \\
Nausea \& vomiting & \\
Signs & 2 \\
RIF tenderness & 1 \\
Rebound tenderness & 1 \\
Fever & \\
Labs & 2 \\
Leukocytosis & 1 \\
Shift of WBC to left & 10 \\
Total
\end{tabular}

Guidelines for management according to the total score: $<4$, probability of acute appendicitis (AA) unlikely; 4-7, AA suspected; >7, definite AA. $\mathrm{RIF}$, right iliac fossa; WBC, white blood cell count. 
Volume 33, Number 6, 2017

day. Histopathology findings on the surgical cases were collected and correlated with the scores. Scores were tabulated, and the sensitivities, specificities, positive and NPVs, and positive and NLRs were calculated. Also, the area under the receiver operating characteristic (ROC) curves were obtained using IBM SPSS Statistics ver. 21.0 (IBM Co., Armonk, NY, USA) and MedCalc ver. 15 (MedCalc, Ostend, Belgium).

\section{RESULTS}

The mean \pm standard deviation (SD) of the patients' ages in our study group was $32 \pm 10$ years old. Of the participants, $66 \%$ were male patients, and of the 100 patients who underwent surgery,

Table 3. Acute inflammatory response scoring system

\begin{tabular}{lc}
\hline Diagnosis & Score \\
\hline Vomiting & 1 \\
Pain in RIF & 1 \\
Abdominal defense & \\
Low & 1 \\
Mild & 2 \\
Severe & 3 \\
Temperature $>38.5^{\circ} \mathrm{C}$ & 1 \\
Segmented neutrophils & \\
$70 \%-84 \%$ & 1 \\
$\geq 85 \%$ & 2 \\
Leukocytes $\left(\times 10^{9} /\right.$ L) & \\
$10.0-14.9$ & 1 \\
$\geq 15.0$ & 2 \\
CRP (g/L) & \\
$10-49$ & 1 \\
$\geq 50$ & 2 \\
Total & \\
\hline
\end{tabular}

AIR: sum 0-4, low probability; sum 5-8, mild probability; sum 9-12, high probability.

RIF, right iliac fossa; CRP, C-reactive protein.
$88 \%$ were positive for appendicitis on the histopathological report. At the optimal cutoff point of $>8$ for the RIPASA scoring system, the sensitivity and the specificity were $93.18 \%$ and $91.67 \%$, respectively. Also, at the optimal cutoff point of $>7$ for the Alvarado scoring system, the sensitivity and the specificity were $78.41 \%$ and $100 \%$, respectively, while at optimal cutoff point of $>4$ for the AIR scoring system, the sensitivity and the specificity were 78.41\% and 91.67\%, respectively. The PPVs and NPVs and the positive and NLRs of the 3 scoring systems are presented in Table 4. According to the ROC curve, the area under the curve (AUC) was 0.981 for the RIPASA scoring system, which is greater than the AUC of 0.906 for the Alvarado scoring system and the AUC of 0.867 for the AIR scoring system (Fig. 1). The difference in the

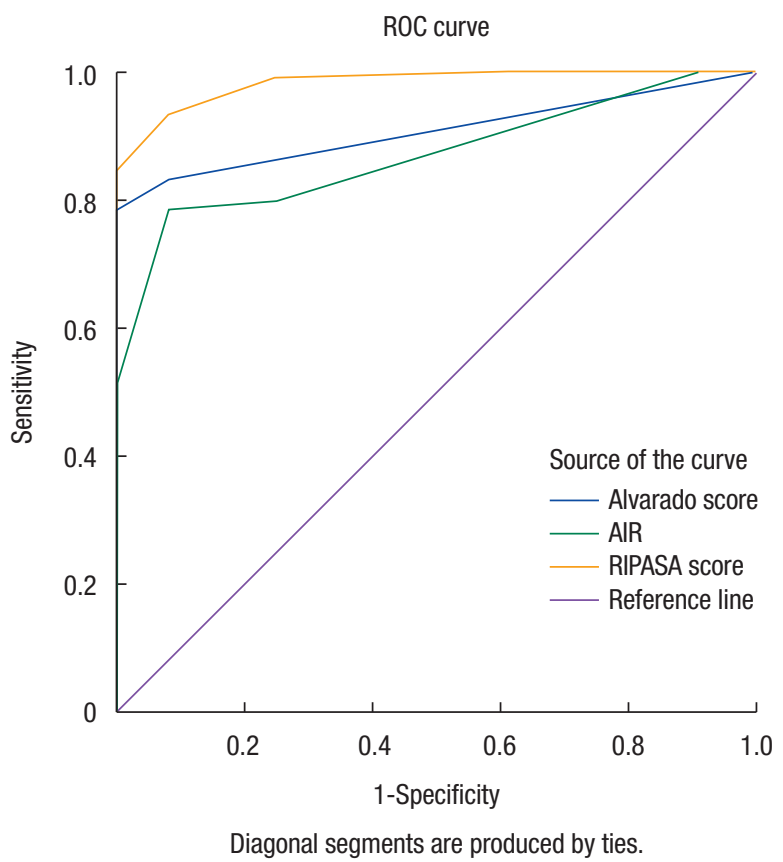

Fig. 1. The receiver operating characteristic (ROC) curves demonstrate the sensitivity vs. specificity of the Alvarado, Raja Isteri Pengiran Anak Saleha Appendicitis (RIPASA), and acute inflammatory response (AIR) scoring systems in the diagnoses of appendicitis.

Table 4. Diagnostic predictive values with individual RIPASA, Alvarado, and AIR scoring systems

\begin{tabular}{lccc}
\hline Statistic & AlR score $(>4)$ & Alvarado score $(>7)$ & RIPASA score $(>8)$ \\
\hline Sensitivity (\%) & $78.41(68.4-86.5)^{\mathrm{a}}$ & $78.41(68.4-86.51)^{\mathrm{a}}$ & $93.18(85.7-97.5)^{\mathrm{a}}$ \\
Specificity (\%) & $91.67(61.5-99.8)^{\mathrm{a}}$ & $100(73.5-100)^{\mathrm{a}}$ & $91.67(61.5-99.8)^{\mathrm{a}}$ \\
Positive likelihood ratio & 9.41 & - & 11.18 \\
Negative likelihood ratio & 0.24 & 0.22 & 0.074 \\
Positive predictive value (\%) & 98.57 & 100 & 98.80 \\
Negative predictive value (\%) & 36.67 & 38.71 & 64.70 \\
\hline
\end{tabular}

RIPASA, Raja Isteri Pengiran Anak Saleha Appendicitis; AIR, acute inflammatory response.

a95\% Confidence interval. 
AUCs of $11.3 \%$ between the RIPASA and the AIR scoring systems was significant $(\mathrm{P}=0.0020)$, as was the difference in the AUCs of $7.48 \%$ between the RIPASA and the Alvarado scoring systems ( $\mathrm{P}$ $=0.0026)$. The difference in the AUCs of 3.84\% between the AIR and the Alvarado scoring systems was not significant $(\mathrm{P}=0.165)$.

\section{DISCUSSION}

Surgery for AA is one of the most common emergent procedures in general surgery. The overall lifetime risks of developing AA are $8.6 \%$ and $6.7 \%$ for males and females, respectively [14]; lifetime risks of undergoing an appendectomy are around $12 \%$ and $23 \%$ in males and females, respectively [15]. A deferral in playing out an appendectomy with a specific end goal to enhance diagnostic precision leads to the danger of appendicular perforation and infection, which enhances the morbidity and the mortality [11]. The inverse is also valid, where with lessened demonstrative accuracy, the negative or unessential appendectomy rate is expanded, and this is described to be roughly $20 \%-40 \%$ [16]. Diagnostic modalities such as ultrasonography or CT imaging in developing countries may raise the cost of healthcare considerably. Unselective use of CT may lead to early appendicitis and needless appendectomies involving patients whose appendicitis might have been improved naturally through the use of antibiotics [17].

The Alvarado scoring system has been the most famous clinical one since its introduction in 1986. It has been shown to have high validity when applied to populations in the United States and Europe. Subsequently, when this scoring system was applied to Asian populations, it revealed a moderately lower specificity and sensitivity in the diagnosis of AA [7, 18-20]. The AIR scoring system is another clinical criterion used for AA diagnosis and has been well known in validation studies during the last decade [21, 22]. Recently, a new scoring system, the RIPASA scoring system, was developed. This new system, which was exclusively designed for use with Asian populations, is broader and simpler and consists of seventeen items and an additional parameter $[5,6]$.

Our study compared the sensitivities, the specificities, the PLRs, the NLRs, the PPVs, and the NPVs between the RIPASA, Alvarado, and AIR scoring systems. The true positive rate (sensitivity) is the proportion of actual positives that are appropriately recognized; this is the percentage of sick people who are properly diagnosed as having appendicitis. The true negative rate (specificity) is the proportion of negatives that are properly recognized; this is the percentage of healthy people who are correctly recognized as not having appendicitis [12]. The PPV and the NPV are the proportion of patients with positive criteria who really have the disease and the proportion of patients with negative criteria who are actually free of the disease, respectively. The likelihood ratio (LR) demonstrates how likely other people are to get positive criteria if they have the disease compared with a person without disease. The PLR is usually a number greater than one, and the NLR ratio is usually a number smaller than one [23-25].
In the present study, the sensitivity of the RIPASA score was significantly better than those of the Alvarado and the AIR scores. The order of the sensitivities for the same population in these scoring system is $93.18 \%$ with a RIPASA score $>8,78.41 \%$ with an Alvarado score $>7$, and $78.41 \%$ with an AIR score $>4$. The specificity of the Alvarado score was more than those of the RIPASA and the AIR scores. In the scott study, an AIR score of 5 or more demonstrated high sensitivities for intermediate and high risk patients with appendicitis (90\%) and for patients with advanced appendicitis (98\%) [26]. In the Chong et al. study [27], the RIPASA score at a cutoff threshold total score of 7.5 was a better diagnostic scoring system than the Alvarado score for the diagnosis of AA at singapore local hospital.

In the study of Memon et al. [28] in an Indian population, the sensitivity and the specificity of the Alvarado scoring system were found to be $93.5 \%$ and $80.6 \%$, respectively. The positive and the NPVs were $92.3 \%$ and $83.3 \%$, respectively. In the present study, the Alvarado scoring system's sensitivity and NPV were less than those mentioned above while the specificity and the PPV were more. Based on these results, the Alvarado score can be used effectively to reduce the incidence of negative appendectomies.

The area under the ROC curve with the RIPASA scoring system was significantly larger than it was with the Alvarado and the AIR scoring systems. The RIPASA score is fast and perfect in categorizing patients with suspected appendicitis and reduces the need for diagnostic imaging. Overall, a higher sensitivity, NPV, and PLR and a lower NLR indicate that the RIPASA score is a much better diagnostic tool than the Alvarado or the AIR score for the diagnosis of AA in Asian people.

The RIPASA score is a useful tool for the diagnosis of AA in Asian populations, as it contains simple parameters that include clinical history, examinations, and 2 simple blood tests. Thus, upon seeing patients with right iliac fossa pain, the operating surgeon can make a quick decision by using the RIPASA scoring system, with a score $>8$ indicating a need for surgery.

\section{CONFLICT OF INTEREST}

No potential conflict of interest relevant to this article was reported.

\section{ACKNOWLEDGMENTS}

The authors would like to thank Shiraz University of Medical Sciences, Shiraz, Iran, and the Center for Development of Clinical Research of Nemazee Hospital for their support. We also thank Dr. Nasrin Shokrpour for editorial assistance. The present article was extracted from the thesis written by Dr. Navid Zadebagheri and was financially supported by Shiraz University of Medical Sciences grants no 93-01-38-8861. 


\section{REFERENCES}

1. Di Saverio S, Birindelli A, Kelly MD, Catena F, Weber DG, Sartelli $\mathrm{M}$, et al. WSES Jerusalem guidelines for diagnosis and treatment of acute appendicitis. World J Emerg Surg 2016;11:34.

2. Zouari M, Jallouli M, Louati H, Kchaou R, Chtourou R, Kotti A, et al. Predictive value of $\mathrm{C}$-reactive protein, ultrasound and $\mathrm{Al}$ varado score in acute appendicitis: a prospective pediatric cohort. Am J Emerg Med 2016;34:189-92.

3. Golden SK, Harringa JB, Pickhardt PJ, Ebinger A, Svenson JE, Zhao YQ, et al. Prospective evaluation of the ability of clinical scoring systems and physician-determined likelihood of appendicitis to obviate the need for CT. Emerg Med J 2016;33:458-64.

4. Liu W, Wei Qiang J, Xun Sun R. Comparison of multislice computed tomography and clinical scores for diagnosing acute appendicitis. J Int Med Res 2015;43:341-9.

5. Chong CF, Adi MI, Thien A, Suyoi A, Mackie AJ, Tin AS, et al. Development of the RIPASA score: a new appendicitis scoring system for the diagnosis of acute appendicitis. Singapore Med J 2010;51:220-5.

6. Butt MQ, Chatha SS, Ghumman AQ, Farooq M. RIPASA score: a new diagnostic score for diagnosis of acute appendicitis. J Coll Physicians Surg Pak 2014;24:894-7.

7. Alvarado A. A practical score for the early diagnosis of acute appendicitis. Ann Emerg Med 1986;15:557-64.

8. Kollár D, McCartan DP, Bourke M, Cross KS, Dowdall J. Predicting acute appendicitis? A comparison of the Alvarado score, the Appendicitis Inflammatory Response Score and clinical assessment. World J Surg 2015;39:104-9.

9. Bozorgi F, Alvandipour M, Montazer SH, Golikhatir I, Khodabakhsh H, Karami M. Diagnostic value of hyperbilirubinemia in acute appendicitis. J Mazandaran Univ Med Sci 2016;26:27-33.

10. Singla A, Singla S, Singh M, Singla D. A comparison between modified Alvarado score and RIPASA score in the diagnosis of acute appendicitis. Updates Surg 2016;68:351-5.

11. N N, Mohammed A, Shanbhag V, Ashfaque K, S A P. A Comparative Study of RIPASA Score and ALVARADO Score in the diagnosis of acute appendicitis. J Clin Diagn Res 2014;8:NC03-5.

12. Mán E, Simonka Z, Varga A, Rárosi F, Lázár G. Impact of the Alvarado score on the diagnosis of acute appendicitis: comparing clinical judgment, Alvarado score, and a new modified score in suspected appendicitis: a prospective, randomized clinical trial. Surg Endosc 2014;28:2398-405.

13. Von-Mühlen B, Franzon O, Beduschi MG, Kruel N, Lupselo D. AIR score assessment for acute appendicitis. Arq Bras Cir Dig
2015;28:171-3.

14. Humes DJ, Simpson J. Acute appendicitis. BMJ 2006;333:530-4.

15. Davies HO, Alkhamesi NA, Dawson PM. Peritoneal fluid culture in appendicitis: review in changing times. Int J Surg 2010;8:426-9.

16. Kalan M, Talbot D, Cunliffe WJ, Rich AJ. Evaluation of the modified Alvarado score in the diagnosis of acute appendicitis: a prospective study. Ann R Coll Surg Engl 1994;76:418-9.

17. Livingston EH, Woodward WA, Sarosi GA, Haley RW. Disconnect between incidence of nonperforated and perforated appendicitis: implications for pathophysiology and management. Ann Surg 2007;245:886-92.

18. Owen TD, Williams H, Stiff G, Jenkinson LR, Rees BI. Evaluation of the Alvarado score in acute appendicitis. J R Soc Med 1992;85: $87-8$.

19. Jang SO, Kim BS, Moon DJ. Application of alvarado score in patients with suspected appendicitis. Korean J Gastroenterol 2008; 52:27-31.

20. Khan I, ur Rehman A. Application of alvarado scoring system in diagnosis of acute appendicitis. J Ayub Med Coll Abbottabad 2005;17:41-4.

21. Andersson M, Andersson RE. The appendicitis inflammatory response score: a tool for the diagnosis of acute appendicitis that outperforms the Alvarado score. World J Surg 2008;32:1843-9.

22. de Castro SM, Ünlü C, Steller EP, van Wagensveld BA, Vrouenraets $\mathrm{BC}$. Evaluation of the appendicitis inflammatory response score for patients with acute appendicitis. World J Surg 2012;36: 1540-5.

23. Altman DG, Bland JM. Diagnostic tests. 1: sensitivity and specificity. BMJ 1994;308:1552.

24. Sedighi I. Interpretation of diagnostic tests: likelihood ratio vs. predictive value. Iran J Pediatr 2013;23:717.

25. Kharbanda AB, Monuteaux MC, Bachur RG, Dudley NC, Bajaj L, Stevenson MD, et al. A clinical score to predict appendicitis in older male children. Acad Pediatr 2017;17:261-6.

26. Scott AJ, Mason SE, Arunakirinathan M, Reissis Y, Kinross JM, Smith JJ. Risk stratification by the appendicitis inflammatory response score to guide decision-making in patients with suspected appendicitis. Br J Surg 2015;102:563-72.

27. Chong CF, Thien A, Mackie AJ, Tin AS, Tripathi S, Ahmad MA, et al. Comparison of RIPASA and Alvarado scores for the diagnosis of acute appendicitis. Singapore Med J 2011;52:340-5.

28. Memon ZA, Irfan S, Fatima K, Iqbal MS, Sami W. Acute appendicitis: diagnostic accuracy of Alvarado scoring system. Asian J Surg 2013;36:144-9. 